\title{
SO SÁNH LƯợNG HƠI NƯỚC TÍCH TỤ TÍNH BẰNG GNSS VÀ BÀ̀NG BÓNG THÁM KHÔNG TẠI TRẠM TÂN SƠN HÒA, THÀNH PHỐ HỒ CHÍ MINH
}

\author{
HUỲNH NGUYẼN ĐịNH QUÓC(1) \\ NGUYẼ̃N NGỌC LÂU(2) \\ ${ }^{(1)}$ Trường Đại học TN\&MT TP HCM \\ ${ }^{(2)}$ Trường ĐHBK TP HCM
}

\section{Tóm tắt:}

Lượng hơ nước tích tụ PWV (Precipitable Water Vapor) trong khí quyển rất cần thiết trong công tác dụ̣ báo thời tiết. Việc xác định chỉ số $P W V$ một cách chính xác hiện nay đang là vấn đề được nhiều người quan tâm trong lĩnh vực khí tượng thủy văn. Trong bài báo này, chúng tôi trình bày thuật toán xác định chỉ số PWV và kết quả so sánh giá trị PWV từ dữ liệu bóng thám không và từ dữ liệu GNSS (Global Navigation Satellite System) tại trạm Tân Sơn Hòa TP.HCM. Độ lệch giữa các kết quả PWV nhỏ hơn 1.2mm. Ngoài ra giá trị PWV thay đổi phù hợp với thời tiết thay đổi trong ngày khảo sát.

\section{Giới thiệu}

Lượng hơi nước tích tụ PWV là lượng hơi nước tại một điểm trên bề mặt trái đất được biểu diễn là độ cao của một cột chất lỏng tương đương. Việc xác định chỉ số PWV một cách chính xác hiện nay đang là vấn đề được nhiều người quan tâm trong lĩnh vực khí tượng thủy văn. Hơi nước khí quyển là một thành phần quan trọng trong sự hình thành của mây, mưa và thời tiết khắc nghiệt. Vì vậy, việc xác định chỉ số PWV là một mục tiêu cơ bản của khí tượng học.

Để xác định chỉ số PWV, hiện nay có nhiều hệ thống đo các tham số khí tượng, chẳng hạn dùng hệ thống máy phóng xạ kế hơi nước, hệ thống bóng thám không vô tuyến, hệ thống đo khí tượng ngay tại bề mặt Trái Đất, hệ thống đo khí tượng bằng bộ phận cảm biến từ xa trên mặt đất (Ground-based remote sensors), hệ thống đo khí tượng từ phi cớ, khinh khí cầu (aircraft)... Một kỹ thuật mới được phát triển gần đây là dùng tín hiệu GNSS để đo hệ số PWV thông qua độ trễ của tín hiệu khi đi qua tầng đối lưu. Qua nghiên cứu của một số tác giả $[5,7,8]$ cho thấy việc xác định PWV từ GNSS phù hợp với kết quả xác định PWV từ hệ thống bóng thám không vô tuyến khoảng 1-2mm. Tại Việt Nam, theo tài liệu $[1,2]$ việc xác định chỉ số PWV từ dữ liệu GNSS phù hợp với kết quả thu được từ ảnh vệ tinh MODIS từ $3-5 \mathrm{~cm}$. Ngoài ra, kết quả hệ số PWV từ thực nghiệm đo GPS cũng tương tự như hệ số PWV từ việc đo các hệ số áp suất, độ ẩm, nhiệt độ tại trạm DON1.

Trong bài báo này, chúng tôi trình bày thuật toán xác định chỉ số PWV từ dữ liệu bóng thám không thông qua các tham số nhiệt độ, áp suất, độ ẩm tương đối... của tầng đối lưu trong phần 2. Ngoài ra, sử dụng dữ liệu GNSS tính toán hệ số PWV trình bày trong phần 3. Phần 4 là kết quả so sánh độ chính xác của hai hệ thống bóng thám không và GPS tại TP.HCM. 
2. Thuật toán xác định chỉ số hơi nước tích tụ PWV từ dữ liệu bóng thám không vô tuyến

\subsection{Thuật toán xác định hệ số $P W V$ của Y. Liu [4]}

Hệ số IWV có thể tính thông qua hệ số PWV [4]:

$$
P W V=\frac{I W V}{\rho}=\frac{\int \rho_{v} \cdot d h}{\rho}
$$

Trong đó: $p_{v}$ - mật độ hơi nước

$\mathrm{h}$ - độ cao theo phương thẳng đứng của tầng đối lưu

$\mathrm{p}$ - hằng số mật độ nước

Theo phương trình trạng thái khí, mật độ hơi nước $p_{v}$ được tính thông qua biểu thức sau:

$$
\rho_{v}=\frac{e_{\mathrm{w}}}{R_{v} \cdot T}=\frac{R H \cdot \exp \left(-37,2465+0,213166 \cdot T-2,256908 \cdot 10^{-4} \cdot T^{2}\right)}{R_{v} \cdot T}
$$

Trong đó: $R_{v}=461.525 . \mathrm{K}^{-1} \cdot \mathrm{kg}^{-1}$ - hằng số khí đặt biệt của hơi nước

T - nhiệt độ (tính theo độ Kelvin)

RH - độ ẩm tương đối (\%)

T - nhiệt độ (tính theo độ Kelvin)

Áp suất hơi nước tính được rời rạc từ dữ liệu thám không vô tuyến dựa vào nhiệt độ và độ ẩm tương đối nhận được ở những độ cao khác nhau, gây ra một sự tách biệt của khí quyển tạo thành nhiều lớp. Giả sử mật độ hơi nước biến đổi tuyến tính trong mỗi lớp, giá trị PWV có thể được xấp xỉ bằng:

$$
P W V=\frac{1}{\rho} \sum\left(h_{j+1}-h_{j}\right) \cdot\left(\rho_{v}^{j+1}+\rho_{v}^{j}\right) / 2
$$

Trong đó: $h_{j+1}$ và $h_{j}$ là độ cao đỉnh và đáy của mỗi lớp độ cao

2.2. Thuật toán xác định hệ số PWV của Văn phòng Dịch vụ Dự báo Thời Tiết Quốc gia của Mỹ (National Weather Service Weather Forecast Office-NWSWFO)

Việc xác định chỉ số PWV từ bóng thám không cũng dựa vào các yếu tố khí tượng nhận được trong quá trình bóng thời tiết di chuyển trong khí quyển như sau: [6]

Sức trương hơi nước của không khí ẩm e

$$
e=f_{p} \cdot e_{w}=\left(1,0016+3,15 \cdot 10^{-6} \cdot P-\left[\frac{0,074}{P}\right]\right) \cdot\left(6,112 \cdot \exp \left[\frac{17,67 \cdot T_{d}}{T_{d}+243,5}\right]\right)
$$


Trong đó: $\mathrm{P}$ - áp suất không khí (hPa)

$\mathrm{T}_{\mathrm{d}}$ - nhiệt độ điểm sương $\left({ }^{\circ} \mathrm{C}\right)$.

Mật độ hơi nước vd (vapor density)

$$
v d=\left[\left(\frac{e}{R_{v} \cdot T}\right)\right] \cdot 10^{5}
$$

Trong đó: Rv = 461.5 (J/K/kg): hệ số hơi nước không đổi

T - nhiệt độ (Kelvin)

Giá trị hệ số hơi nước tích tụ PWV

$$
p w v=\sum p w=\sum v d^{*} \cdot h \cdot 10^{-4}
$$

Với: $v d^{*}$ - giá trị trung bình của mật độ hơi nước $\left(\mathrm{g} / \mathrm{m}^{3}\right)$

$\mathrm{h}$ - chiều dày của lớp độ cao $(\mathrm{m})$

Trong bài báo này, chúng tôi sử dụng cả 2 thuật toán tính chỉ số PWV từ bóng thám không vô tuyến của Liu [4] và của NWSWFO [6] để so sánh với PWV từ dữ liệu GNSS.

\section{Thuật toán xác định chỉ số hơi nước tích tụ PWV từ dữ liệu đo GNSS.}

Độ trễ của tín hiệu xảy ra ở tầng đối lưu nhận được từ việc xử lý GNSS thường được chia thành hai thành phần khác nhau bao gồm: độ trễ khô thiên đỉnh (ZHD - Zenith Hydrostatic Delay) và độ trễ ướt thiên đỉnh (ZWD - Zenith Wet Delay). Độ trễ đối lưu dọc theo đường truyền tín hiệu GPS được biểu diễn dưới dạng sau:

$$
T=m_{d}(\vartheta) \cdot Z H D+m_{w}(\vartheta) \cdot Z W D
$$

Trong đó: $m_{d}(\vartheta)$ - hàm ánh xạ khô

$$
\begin{gathered}
m_{w}(\vartheta) \text { - hàm ánh xạ ướt } \\
\vartheta \text { - góc cao vệ tinh }
\end{gathered}
$$

Trong thực tế, hàm ánh xạ khô và hàm ánh xạ ướt rất gần nhau khi góc cao vệ tinh trên $15^{\circ}$ nên thường tính xấp xỉ:

$$
T=m_{d}(\vartheta) \cdot Z H D+m_{w}(\vartheta) \cdot Z W D=m(\vartheta) \cdot T Z D
$$

Trong đó: $m_{d}(\vartheta)=m_{w}(\vartheta)=m(\vartheta)$ hàm ánh xạ khô hay ướt.

TZD: độ trễ đối lưu thiên đỉnh toàn bộ (TZD: Toposhere Zenith Delay)

Từ đó: $\quad T Z D=Z H D+Z W D$

Mặt khác, mối quan hệ giữa PWV với ZWD được thể hiện:

$$
P W V=\frac{Z W D}{Q}=\Pi . Z W D=\Pi .(T Z D-Z H D)
$$


Trong đó $\Pi$ có giá trị trung bình 0.159 và được tính theo nhiệt độ đo ở bề mặt.

Để xác định độ trễ đối lưu thiên đỉnh toàn bộ TZD từ trị đo pha GNSS, chúng tôi sử dụng phương pháp định vị tuyệt đối [3]. Còn chỉ số ZHD được tính chính xác đến $\mathrm{mm}$ theo các trị đo khí tượng bề mặt [8].

Độ chính xác xác định TZD bằng GNSS khi có sử dụng các sản phầm của IGS khoảng $10 \mathrm{~mm}$ [3]. Do đó ta có thể ước tính độ chính xác xác định PWV bằng GNSS như sau:

$$
m_{P W V}=\Pi \sqrt{m_{T Z D}^{2}+m_{Z H D}^{2}}=0.159 \sqrt{10^{2}+2^{2}}=1.6 \mathrm{~mm}
$$

\section{Phương pháp xác định độ trễ đối lưu thiên đỉnh TZD}

Phương trình trị đo pha GPS trên tần số $L_{1}$ và $L_{2}$ (từ vị trí máy i đến vệ tinh thứ $k$ )

$$
\begin{gathered}
\varphi_{i, 1}^{k}=\frac{f_{1}}{c} \rho_{i}^{k}+f_{1}\left(d t_{i}-d T^{k}\right)+N_{i, 1}^{k}+\frac{f_{1}}{c} T_{i}^{k}-\frac{f_{1}}{c} I_{i, 1}^{k}+d_{o r b 1}^{k}+d_{m u l 1}^{k}+\varepsilon_{1}^{k} \\
\varphi_{i, 2}^{k}=\frac{f_{2}}{c} \rho_{i}^{k}+f_{2}\left(d t_{i}-d T^{k}\right)+N_{i, 2}^{k}+\frac{f_{2}}{c} T_{i}^{k}-\frac{f_{2}}{c} I_{i, 2}^{k}+d_{o r b 2}^{k}+d_{m u l t 2}^{k}+\varepsilon_{2}^{k}
\end{gathered}
$$

Trong đó:

$\varphi_{i, 1}^{k} ; \varphi_{i, 2}^{k} \quad$ là trị đo pha trên $\mathrm{L}_{1}$ và $\mathrm{L}_{2}$

$\rho_{i}^{k} \quad$ là khoảng cách hình học giữa máy thu và vệ tinh.

$\left(X^{k}, Y^{k}, Z^{k}\right)$ là tọa độ vệ tinh (nhận từ sản phẩm bản lịch final của IGS)

$\left(\mathrm{X}_{\mathrm{i}}, \mathrm{Y}_{\mathrm{i}}, \mathrm{Z}_{\mathrm{i}}\right)$ là tọa độ máy thu

$d t_{i} \quad$ là sai số đồng hồ máy thu

$d T^{k} \quad$ là sai số đồng hồ vệ tinh

$N_{i, 1}^{k} ; N_{i, 2}^{k} \quad$ là tham số đa trị trên 2 tần số.

$d_{o r b}^{k} \quad$ là sai số quỹ đạo vệ tinh

$d_{m u l t}^{k}, d_{m u l t 2}^{k}$ là ảnh hưởng của đa đường trên trị đo pha tần số $\mathrm{L}_{1}$ và $\mathrm{L}_{2}$.

$T_{i}^{k} \quad$ là độ trễ đối lưu theo dường truyền tín hiệu $\left(T_{i}^{k}=m(\vartheta) . T Z D\right)$

$I_{i, 1}^{k} ; I_{i, 2}^{k} \quad$ là độ trễ điện ly (theo đường truyền tín hiệu) trên tần số $\mathrm{L}_{1}$ và $\mathrm{L}_{2}$.

$\varepsilon_{1}^{k} ; \varepsilon_{2}^{k} \quad$ là sai số đo của trị đo pha trên 2 tần số.

c = 299792.458km/s là vận tốc của ánh sáng trong chân không

$\mathrm{f}_{1}=1575.42 \mathrm{MHz}$ và $\mathrm{f}_{2}=1227.60 \mathrm{MHz}$ là tần số $\mathrm{GPS}$ trên tín hiệu $\mathrm{L}_{1}$ và $\mathrm{L}_{2}$

Để loại bỏ sai số ảnh hưởng tầng điện ly, thành lập tổ hợp $L_{3}$ cho trị đo pha sóng tải bằng cách kết hợp phương trình trị đo pha $\varphi_{1}$ và $\varphi_{2}$ (chuyển từ đơn vị chu kỳ pha sang đơn vị m): $L_{3}^{k}=\alpha_{1} \varphi_{i, 1}^{k}-\alpha_{2} \varphi_{i, 2}^{k}$

$$
\lambda_{1} L_{i, 3}^{k}=\Phi_{i, 3}^{k}=\rho_{i}^{k}+c\left(d t_{i}-d T^{k}\right)+\lambda_{1} N_{3}+T Z D(m(\vartheta))+\lambda_{1} d_{o r b 3}+\lambda_{1} d_{m u l t 3}+\lambda_{1} \varepsilon_{3}
$$


Trong đó:

$$
\begin{gathered}
\alpha_{1}=\frac{f_{1}^{2}}{f_{1}^{2}-f_{2}^{2}} ; \alpha_{2}=\frac{f_{2}^{2}}{f_{1}^{2}-f_{2}^{2}} \\
N_{3}=\alpha_{1} N_{i, 1}^{k}-\alpha_{2} N_{i, 2}^{k} \quad \text { là tham số đa trị của trị đo tổ hợp } \mathrm{L}_{3} \\
d_{\text {orb3 }}=d_{\text {orb1 }}-d_{\text {orb2 }} \text { là sai số quỹ đạo vệ tinh trị đo tổ hợp } \mathrm{L}_{3} \\
d_{\text {mult3 }}=d_{\text {mult1 }}-d_{\text {mult2 }} \text { là ảnh hưởng của hiện tượng đa đường trị đo tổ hợp } \mathrm{L}_{3} \\
\varepsilon_{3}=\varepsilon_{1}-\varepsilon_{2} \quad \text { là sai số đo của trị đo } \mathrm{L}_{3} \\
\lambda_{1}=1575.42 \mathrm{MHz}
\end{gathered}
$$

Việc lấy hiệu giữa các trị đo tổ hợp $L_{3}$ (giữa 2 vệ tinh và một máy thu) sẽ khử sai số của đồng hồ máy thu

$$
\Phi_{i, 3}^{l-k}=\Phi_{i, 3}^{l}-\Phi_{i, 3}^{k}=\left(\rho_{i}^{l}-\rho_{i}^{k}\right)+c\left(d T^{l}-d T^{k}\right)+\lambda_{1}\left(N_{3}^{l}-N_{3}^{k}\right)+\operatorname{TZD}\left(m\left(\vartheta^{l}\right)-m\left(\vartheta^{k}\right)\right)
$$

Ngoài ra, sai số do sai số đồng hồ vệ tinh $\left(d T-d T^{k}\right)$, và giá trị $\rho_{i}^{l}-\rho_{i}^{k}$ được tính toán chính xác và loại ra khỏi phương trình (13) khi sử dụng sản phẩm của tổ chức IGS (International GNSS Service) cung cấp tọa độ vệ tinh với độ chính xác sai số quỹ đạo vệ tinh $5 \mathrm{~cm}$ và sai số đồng hồ vệ tinh 0.1 nano giây.

Trong phương trình (14) trên các ẩn số còn lại bao gồm tham số đa trị $\left(N_{3}^{l}-N_{3}^{k}\right)$ là hằng số, và độ trễ đối lưu thiên đỉnh TZD thay đổi theo không gian và thời gian. Để xử lý tham số này, chúng tôi sử dụng bộ lọc Kalman, trong đó mô hình hóa tham số TZD bằng quá trình bước ngẫu nhiên. Sau khi tính giá trị TZD, sử dụng phương trình (10) để tính giá trị PWV. (Xem hình 1)

Để kiểm tra tính đúng đắn của thuật toán và chương trình, học viên đã chọn xử lý dữ liệu GPS tại trạm đo IGS NTUS - Singapore tại các ngày 16, 17-01-2013 và ngày 18, 19, 20-09-2013 trùng với các ngày thu GNSS khảo sát vì những lý do:

- Là trạm IGS ở gần Việt Nam nên phần nào thể hiện tính chất biến động TZD tương tự như trên lãnh thổ Việt Nam.

- Dữ liệu IGS ở trạm này được thu bằng máy thu GNSS chất lượng cao, tốc độ thu 30s và tọa độ của nó đã biết với tọa độ chính xác trong hệ tọa độ ITRF2008.

- Sản phẩm TZD của trạm này có sẵn cho khoảng thời gian 05 phút/01 giá trị với độ chính xác khoảng $4 \mathrm{~mm}$ [3]

Kết quả từ việc xử lý theo thuật toán và kết quả của IGS được cho ở hình 2. (Xem H.2)

\section{Kết quả so sánh kết quả PWV của hai hệ thống bóng thám không và GNSS tại TP.HCM}

Chúng tôi sử dụng dữ liệu bóng thám không trạm Tân Sơn Hòa TP.HCM để xác định chỉ số PWV trong các ngày 16, 17-01-2013 (đợt 1) và 18, 19 và 20-09-2013 (đợt 2) bên cạnh việc sử dụng dữ liệu khảo sát của trạm đo GNSS tại TP.HCM để xác định chỉ số PWV trong cùng thời gian thả bóng thám không. Kết quả xác định chỉ số PWV từ hai hệ thống 


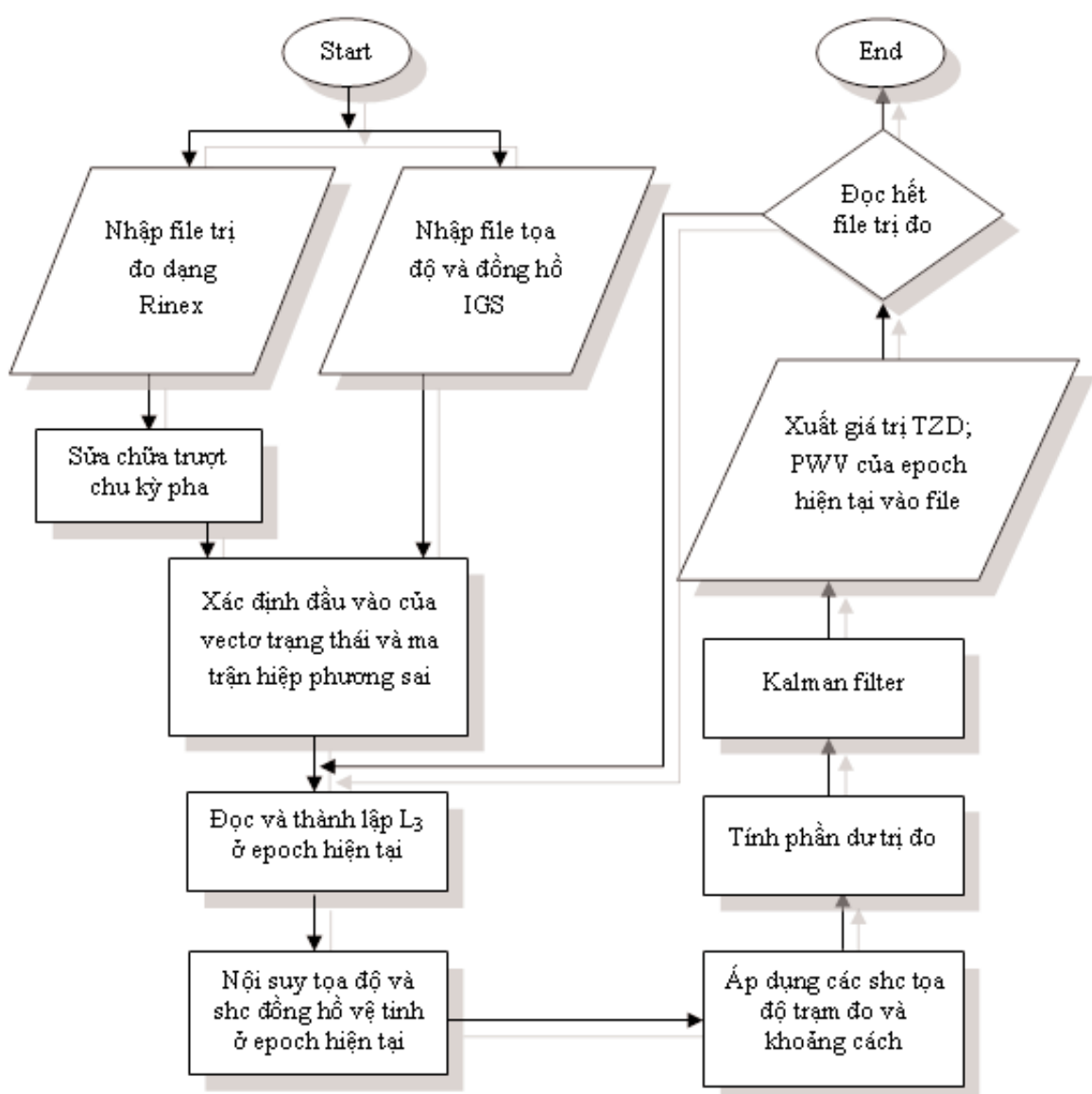

Hình 1: Thuật toán xác định TZD và PWV từ dữ liệu GPS (chấp nhận từ tài liệu [3]) được thể hiện ở hình 3, 4 và bảng 1.

Theo kết quả trong bảng 1 cho thấy giá trị PWV của bóng thám không và GNSS trong hai đợt khảo sát có kết quả tương đồng nhau, độ lệch lớn nhất là $1.2 \mathrm{~mm}$ (trong ngày 19 và 20-09-2013). Mặt khác, giá trị PWV nhận được có sự thay đổi đáng kể, từ 32 - 38mm (đợt 1 ) tăng lên $35-65 \mathrm{~mm}$ (đợt 2). Kết quả này tương đối đáng tin cậy khi trong đợt 1 (ngày 16, 17-01-2013) trời nắng, không mưa, giá trị PWV thấp, dao động trong khoảng 32 - 38mm, còn trong đợt 2 (ngày 18, 19, 20-09-2013) thời tiết âm u, trời mưa, độ ẩm cao, giá trị PWV lớn, dao động trong khoảng $35-65$ mm.

\section{Tóm tắt và kết luận}

Chúng tôi đã trình bày hai thuật toán tính chỉ số PWV từ bóng thám không và từ GNSS, và so sánh chỉ số PWV nhận được từ hai hệ thống trên trong thời gian 16, 17/1/2013 (đợt 1) và 18,19 và 20/09/2013 (đợt 2). Kết quả cho thấy:

- Công thức tính PWV từ bóng thám không của Liu và NWSWFO cho kết quả giống nhau. Tuy nhiên công thức Liu cho kết quả phù hợp với GNSS hơn.

- Độ lệch PWV giữa GNSS và bóng thám không nhỏ hơn $1.2 \mathrm{~mm}$, tương đồng với các kết quả nghiên cứu trên thế giới từ 1-2mm. Đồng thời giá trị PWV nhận được phù hợp với 


\section{Trao đổi - Ý kiến}

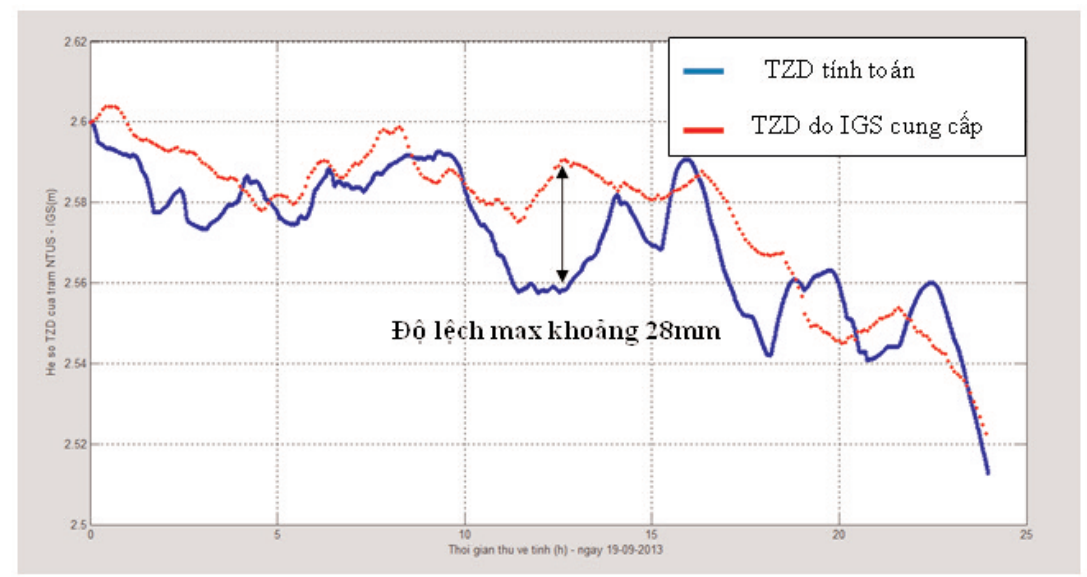

Hình 2: TZD từ thuật toán (chấm xanh) và từ IGS (chấm đỏ) ngày 19-9-2013

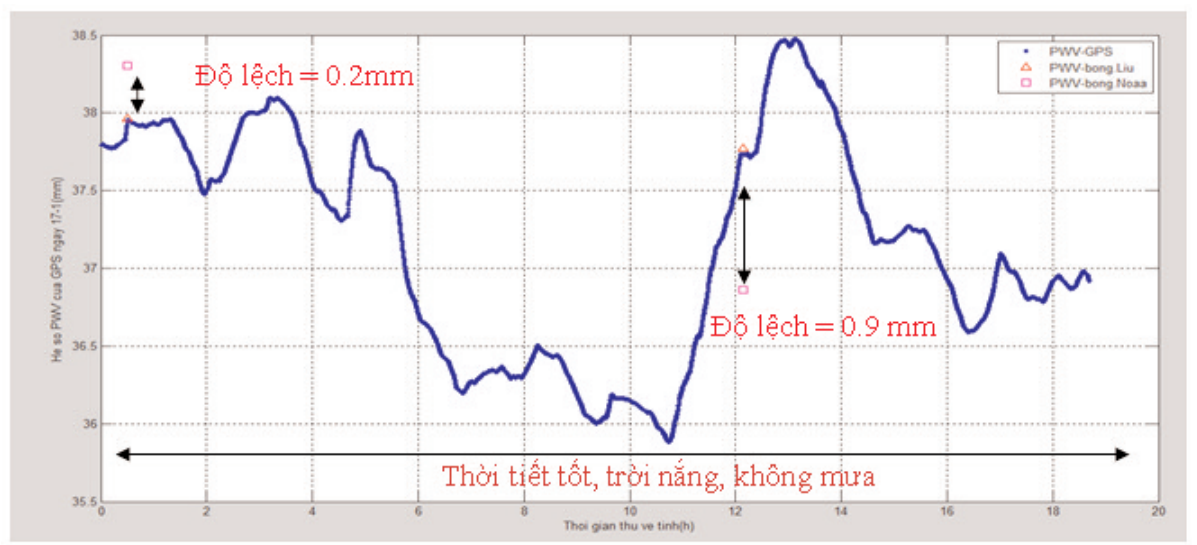

Hình 3: PWV từ dữ liệu đo GNSS (đường xanh) và từ bóng thám không (thuật toán Liu: tam giác và NWSWFO: hình vuông) ngày 17-01-2013

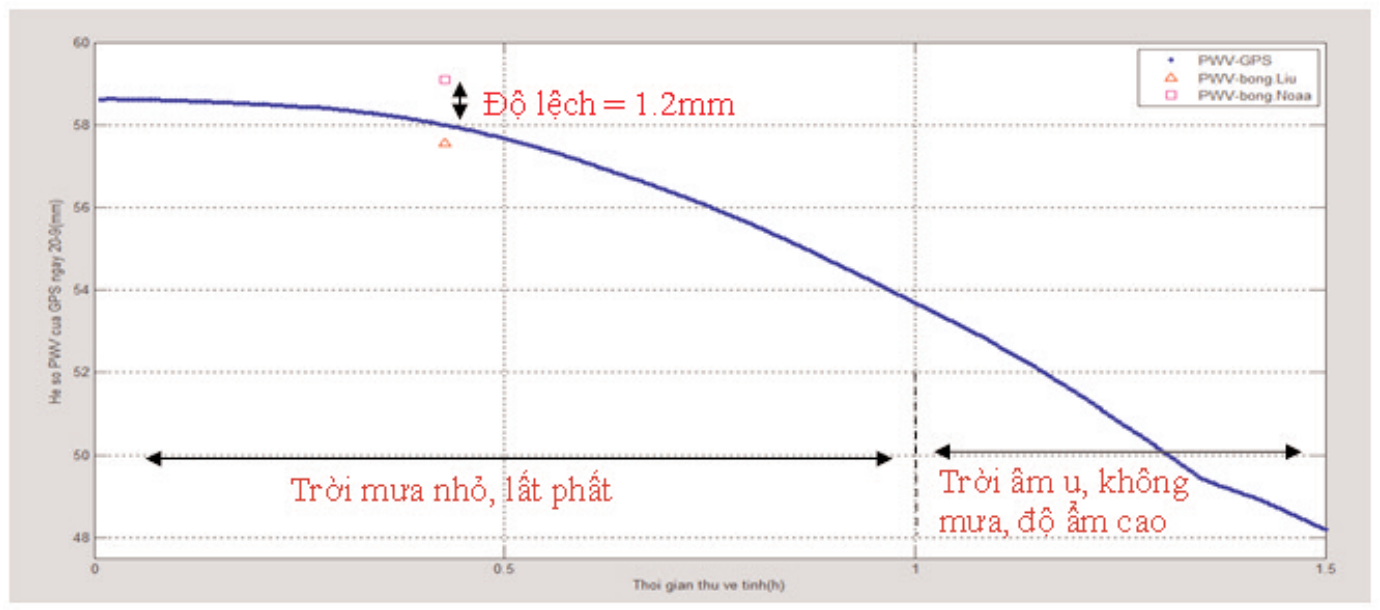

Hình 4: PWV từ dữ liệu đo GNSS (đường xanh) và từ bóng thám không (thuật toán Liu: tam giác và NWSWFO: hình vuông) ngày 20-09-2013 
Bảng 1: Độ lệch giữa PWV từ bóng thám không và GNSS

\begin{tabular}{|c|c|c|}
\hline \multirow{2}{*}{$\begin{array}{c}\text { Thời gian } \\
\text { (UTC) }\end{array}$} & \multicolumn{2}{|c|}{ Độ lệch của hệ số PWV giữa GNSS và } \\
\cline { 2 - 3 } & Liu & NWSWFO \\
\hline $16-01-2013$ & $0.5 \mathrm{~mm}$ & $0.8 \mathrm{~mm}$ \\
\hline \multirow{2}{*}{$17-01-2013$} & $0.2 \mathrm{~mm}$ & $0.4 \mathrm{~mm}$ \\
\hline & $0.2 \mathrm{~mm}$ & $0.9 \mathrm{~mm}$ \\
\hline $18-09-2013$ & $-0.9 \mathrm{~mm}$ & $0.5 \mathrm{~mm}$ \\
\hline $19-09-2013$ & $0.7 \mathrm{~mm}$ & $1.2 \mathrm{~mm}$ \\
\hline $20-09-2013$ & $-0.4 \mathrm{~mm}$ & $1.2 \mathrm{~mm}$ \\
\hline
\end{tabular}

sự thay đổi của thời tiết trong khoảng thời gian khảo sát.

Dựa vào kết quả PWV đạt được giữa bóng thám không và GNSS từ đó có thể cung cấp chúng ta thêm một phương pháp mới trong công tác dự báo thời tiết của ngành khí tượng thủy văn bằng cách sử dụng các hệ thống định vị vệ tinh GNSS trong xử lý bằng định vị tuyệt đối. Ngoài ra, GNSS cung cấp hệ số hơi nước tích tụ PWV trong khí quyển liên tục trong suốt thời gian đo, nhưng chi phí chỉ đầu tư một lần, trong khi hệ thống bóng thám không vô tuyến chỉ cung cấp được một giá trị PWV duy nhất ở mỗi lần thả bóng, chi phí cho mỗi lần thả bóng tốn kém rất nhiều. $\bigcirc$

\section{Tài liệu tham khảo}

[1]. Dương Chí Công, Nguyễn Ngọc Lâu, Hà Minh Hòa, Vũ Thanh Ca, (2009), "Một số kết quả nghiên cứu sử dụng số liệu đo GPS độ chính xác cao để xác định tổng lượng điện tử phương thiên đỉnh (VTEC) và lượng hơi nước tích tụ (PWV) tại Việt Nam", Tuyển tập báo cáo hội nghị khoa học: "Đo đạc và bản đồ Việt Nam vì sự nghiệp xây dựng và bảo vệ tổ quốc", Kỷ niệm 50 năm thành lập ngành đo đạc và bản đồ Việt Nam, Hà Nội, 12/2009, pp. 32-38.

[2]. Hà Minh Hòa và Nguyễn Ngọc Lâu, (2005), "Khả năng sử dụng các dữ liệu thu tín hiệu vệ tinh GPS trên các trạm thu thường trực để xác định độ ẩm không khí ở tầng đối lưu", Tuyển tập Hội thảo Báo cáo Khoa học lần thứ 9 của Viện Khí Tượng Thủy Văn - Bộ tài nguyên Môi trường, Hà Nội 12/2005, pp. 94-101.

[3]. Nguyễn Ngọc Lâu, (2012), "Xác định lượng hơi nước tích tụ (PWV) bằng phương pháp định vị điểm GPS chính xác”, Tạp chí Khí tượng Thủy văn, số 614, pp. 40-44.

[4]. Y.Liu, (2000), "Precision of precipitable water vapor from radiosonde data for GPS solutions", Geomatica, Vol.54, No.2.2000, pp. 171-175.

[5]. A. J. Coster, A. E. Niell, F. S. Solheim, V. B. Mendes, P. C. Toor, K. P. Buchmann, C. A. Upham, (1996), "Measurements of Precipitable Water Vapor by GPS, Radiosondes, and a Microwave Water Vapor Radiometer", Presented at Ion-GPS, Kansas City, Kansas, September 17-20, 1996.

[6]. Precipitable Water Plots, <http://www.crh.noaa.gov/unr/?n=pw>

(Xem tiếp trang 46) 